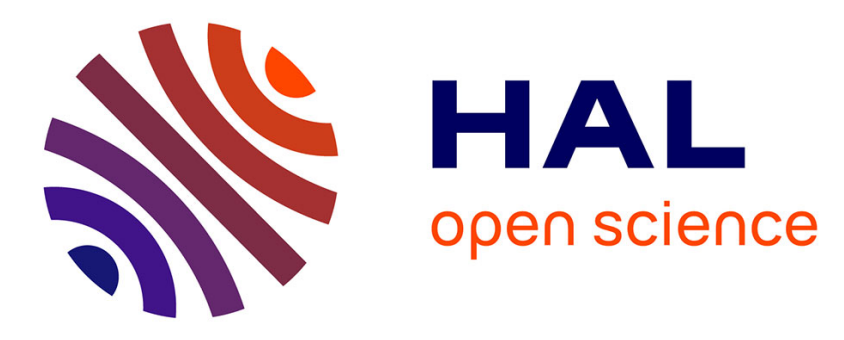

\title{
Computing combustion noise by combining large eddy simulations with analytical models for the propagation of waves through turbine blades
}

Ignacio Duran, Matthieu Leyko, Stéphane Moreau, Franck Nicoud, Thierry Poinsot

\section{To cite this version:}

Ignacio Duran, Matthieu Leyko, Stéphane Moreau, Franck Nicoud, Thierry Poinsot. Computing combustion noise by combining large eddy simulations with analytical models for the propagation of waves through turbine blades. Comptes rendus de l'Académie des sciences. Série IIb, Mécanique, 2013, 341 (1-2), pp.131-140. 10.1016/j.crme.2012.10.012 . hal-00821090

\section{HAL Id: hal-00821090 \\ https://hal.science/hal-00821090}

Submitted on 7 May 2013

HAL is a multi-disciplinary open access archive for the deposit and dissemination of scientific research documents, whether they are published or not. The documents may come from teaching and research institutions in France or abroad, or from public or private research centers.
L'archive ouverte pluridisciplinaire HAL, est destinée au dépôt et à la diffusion de documents scientifiques de niveau recherche, publiés ou non, émanant des établissements d'enseignement et de recherche français ou étrangers, des laboratoires publics ou privés. 


\title{
Computing combustion noise by combining Large Eddy Simulations with analytical models for the propagation of waves through turbine blades
}

\author{
I. Duran ${ }^{\mathrm{a}, \mathrm{b}}, \mathrm{M}_{\text {Leyko }}{ }^{\mathrm{a}}, \mathrm{S}$. Moreau $^{\mathrm{c}}, \mathrm{F}$. Nicoud $^{\mathrm{d}}$, T. Poinsot $^{\mathrm{e}}$ \\ a Snecma, 77550 Moissy-Cramayel, France.$$
{ }^{\mathrm{b}} \text { CERFACS, } 31057 \text { Toulouse, France. }
$$ \\ ' Université de Sherbrooke, Sherbrooke, QC J1K2R1, Canada. \\ ${ }^{\mathrm{d}}$ Université Montpellier II, 34095 Montpellier, France. \\ e CNRS, Institut de mécanique des fluides, Toulouse, France. \\ Received ${ }^{* * * * *}$; accepted after revision +++++ \\ Presented by Ignacio Duran
}

\begin{abstract}
Two mechanisms control combustion noise generation as shown by Marble and Candel [1]: direct noise, in which acoustic waves propagate through the turbine stages and indirect noise, in which vorticity and/or entropy waves generate noise as they are convected through turbine stages. A method to calculate combustion-generated noise has been implemented in a tool called CHORUS. The method uses the Large eddy simulations of the combustion chamber obtained with the unstructured solver AVBP developed at CERFACS [2] and analytical models for the propagation through turbine stages. The propagation models [3] use the compact row hypothesis to write matching conditions between the inlet and the outlet of a turbine stage. Using numerical simulations, the validity of the analytical methods is studied and the errors made quantified.
\end{abstract}

\section{Résumé}

Méthode de calcul du bruit de combustion combinant des simulations aux grandes échelles avec des méthodes analytiques pour la propagation des ondes à travers les étages de turbine. Deux mécanismes sont responsables de la génération de bruit de combustion : le bruit direct, dans lequel les ondes acoustiques se propagent à travers les étages de la turbine et le bruit indirect, dans lequel les ondes de vorticité ou entropiques génèrent du bruit quand elles sont convectées. Une méthode pour calculer le bruit de combustion a été mise en place dans l'outil CHORUS. La méthode utilise des simulations aux grandes échelles (LES) pour la chambre de combustion, avec le code AVBP developpé au CERFACS [2], et des méthodes analytiques pour la propagation des ondes à travers les étages de la turbine. Le modèle est validé avec des simulations numériques.

Key words: Combustion ; Thermoacoustics ; Indirect combustion noise ; Large Eddy Simulation

Mots-clés : Combustion; Thermoacoustique; Bruit de combustion indirect; Simulation aux Grandes Échelles

$\begin{array}{lllll}\text { Article } & \text { submitted } & \text { to } & \text { INCA } & \text { Colloquim }\end{array}$




\section{Introduction}

The contribution of combustion noise to the total acoustic emissions of the aircraft has increased in commercial airplanes as the noise generated by other sources such as jet and fan noise have been reduced. This noise reduction must continue to meet the increasingly restrictive regulations imposed by international organizations such as ICAO.

Two mechanisms of combustion noise generation were identified by Marble and Candle [1]: direct and indirect combustion noise. In the first one, acoustic waves generated in the combustion chamber propagate through turbine stages to the outlet of the engine. In the second mechanism, noise is generated in the turbine stages by entropy waves convected from the combustion chamber. Marble and Candle proposed a simple one-dimensional analytical tool to study the propagation of waves through a nozzle. The two mechanisms of combustion noise generation have been the focus of recent studies $[4,5,6]$, showing that indirect noise cannot be neglected and that the propagation of acoustic and entropy waves should be taken into account to compute correctly the contribution of combustion to the global noise.

Cumpsty and Marble [3] developed an analytical method, similar to the one-dimensional model of Marble and Candel, to study the propagation of waves in a two-dimensional configuration, allowing a more detailed study of the effects of the propagation of waves through the turbine stages. Using this analytical method, a tool named Chorus has been built at CERFACS to perform the post-processing of the numerical simulations performed using AVBP code [2] and the propagation of noise and entropy waves through fixed and rotating blades of the turbine to compute the combustion noise generated at the outlet.

The analytical theory used for the propagation of waves is explained in Section 2 and the methodology to compute combustion noise is discussed in Section 3. A validation of the analytical theory is proposed in Section 4. Conclusions are presented in Section 5.

\section{Analytical model for the propagation of waves through a turbine}

The analytical model proposed by Cumpsty and Marble [3] deals with the propagation of acoustic and entropy waves through turbine stages assuming a two dimensional configuration. Using the compact blade row hypothesis, matching conditions can be written between the inlet and the outlet of the turbine stage. Fig. 1 shows a sketch of the configuration used here: a infinitely thin blade row acts as an interface between two regions of uniform flow. Vector $\overline{\mathbf{w}}$ represents the mean flow velocity (with module $\bar{w}$ and an angle $\bar{\theta}$ with the axial direction), and $\mathbf{k}$ is the wave vector: the direction of propagation of the considered wave (similarly with module $k$ and angle $\nu$ ).

The two-dimensional steady flow upstream and downstream of the blade row can be described with four variables: the modulus of the steady velocity $\bar{w}$, its angle $\bar{\theta}$, the mean pressure $\bar{p}$ and the mean density of the flow $\bar{\rho}$. It is assumed that the steady flow is perturbed by small fluctuations. These fluctuations can be described with four primitive variables: the entropy perturbation $s^{\prime}$, the mean speed perturbation $w^{\prime}$, the pressure fluctuation $p^{\prime}$ and the perturbation on the mean flow angle $\theta^{\prime}$.

The analytical model of Cumpsty and Marble [3] used by Leyko et al. [9] firstly analyses the propagation of the perturbations in the upstream and downstream regions of the blade row to yield the acoustic, entropy and vorticity waves as a function of the primitive variables $s^{\prime} / C p, w^{\prime} / \bar{c}, p^{\prime} / \gamma \bar{p}$ and $\theta^{\prime}$. Matching conditions are finally written for the fluctuating primitive variables between both sides of the blade row.

Email address: ignacio.duran@cerfacs.fr (I. Duran). 


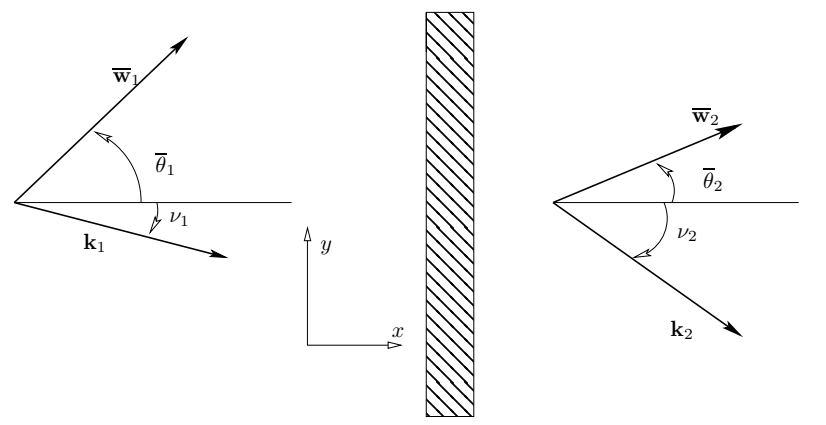

Figure 1. Sketch of the configuration studied

\subsection{Wave decomposition upstream and downstream of the blade row}

The linearized Euler equations (LEE) in a steady uniform flow can be used in the upstream and downstream regions to describe the propagation of the acoustic waves, namely,

$$
\begin{aligned}
& \frac{D}{D t}\left(\frac{\rho^{\prime}}{\bar{\rho}}\right)+\frac{\partial u^{\prime}}{\partial x}+\frac{\partial v^{\prime}}{\partial y}=0 \\
& \frac{D}{D t}\left(u^{\prime}\right)=-\frac{1}{\bar{\rho}} \frac{\partial p^{\prime}}{\partial x} \\
& \frac{D}{D t}\left(v^{\prime}\right)=-\frac{1}{\bar{\rho}} \frac{\partial p^{\prime}}{\partial y} \\
& \frac{D}{D t}\left(\frac{s^{\prime}}{c_{p}}\right)=\frac{D}{D t}\left(\frac{p^{\prime}}{\gamma \bar{p}}-\frac{\rho^{\prime}}{\bar{\rho}}\right)=0
\end{aligned}
$$

where the material derivative is written,

$$
\frac{D}{D t}=\frac{\partial}{\partial t}+\overline{\mathbf{w}} \cdot \nabla=\frac{\partial}{\partial t}+\bar{u} \frac{\partial}{\partial x}+\bar{v} \frac{\partial}{\partial y}
$$

with $\bar{u}=\bar{w} \cos \bar{\theta}$ and $\bar{v}=\bar{w} \sin \bar{\theta}$ the axial and circumferential components of the mean velocity. The fluctuating variables $u^{\prime}$ and $v^{\prime}$ are the velocity perturbations in the $x$ and $y$ directions respectively. They can be related to $w^{\prime}$ and $\theta^{\prime}$ through

$$
\frac{u^{\prime}}{\bar{u}}=\frac{w^{\prime}}{\bar{w}}-\theta^{\prime} \tan \bar{\theta}, \quad \frac{v^{\prime}}{\bar{v}}=\frac{w^{\prime}}{\bar{w}}+\theta^{\prime} / \tan \bar{\theta}
$$

Equations (1)-(4) describe the propagation of the waves in a uniform two-dimensional steady flow. In this region any wave $\phi$ can be written in the form

$$
w^{\phi}=A_{\phi} \exp \left[-i\left(\omega t-\mathbf{k}_{\phi} \cdot \mathbf{x}\right)\right]
$$

where $A_{\phi}$ represents the amplitude, $\omega$ the angular frequency and $\mathbf{k}_{\phi}$ the wave vector associated with the wave, which can be expressed as a combination of the axial and circumferential components,

$$
\mathbf{k}_{\phi} \cdot \mathbf{x}=k_{x, \phi} x+k_{y, \phi} y, \quad \text { with } \quad k_{x, \phi}=k_{\phi} \cos \nu_{\phi} \quad \text { and } \quad k_{y, \phi}=k_{\phi} \sin \nu_{\phi},
$$

where $\nu$ is the angle of the propagating wave, and $k$ the modulus of the wave vector $\mathbf{k}$, as shown in Fig. 1. For simplicity, the wave vector will be scaled with the mean sound speed and the frequency, giving the dimensionless wave vector, $\mathbf{K}=\mathbf{k} \bar{c} / \omega$ (with $\bar{c}$ the mean sound speed). 
Equations (1)-(4) can be used to relate, for each wave, the wave vector with the angular frequency and the mean Mach number.

\subsubsection{Entropy wave}

The entropy wave, $w^{s}=s^{\prime} / C_{p}$, can be written in the wave form using Eq. (7) giving,

$$
w^{s}=\frac{s^{\prime}}{c_{p}}=A_{s} \exp \left[-i\left(\omega t-\mathbf{k}_{s} \cdot \mathbf{x}\right)\right] .
$$

Using Eq. (4), the dispersion equation reads

$$
K_{s} \bar{M} \cos \left(\nu_{s}-\bar{\theta}\right)-1=0 .
$$

The dispersion equation can be used to obtain the axial component of the wave vector (the propagation speed), as a function of the circumferential component, the frequency and the mean flow Mach number and direction.

The entropy wave does not generates pressure or velocity perturbations, therefore, the fluctuation of primitive variables $\left(s^{\prime} / C p, w^{\prime} / \bar{c}, p^{\prime} / \gamma \bar{p}\right.$ and $\left.\theta^{\prime}\right)$ generated by the entropy wave are

$$
\left\{\begin{array}{c}
s^{\prime} / C_{p} \\
w^{\prime} / \bar{c} \\
p^{\prime} / \gamma \bar{p} \\
\theta^{\prime}
\end{array}\right\}=\left\{\begin{array}{l}
1 \\
0 \\
0 \\
0
\end{array}\right\} w^{s} .
$$

\subsubsection{Vorticity wave}

The vorticity wave is written as

$$
\xi^{\prime}=\frac{\partial v^{\prime}}{\partial x}-\frac{\partial u^{\prime}}{\partial y} .
$$

From Eqs. (2) and (3), the equation for the propagation of the vorticity wave reads

$$
\frac{D}{D t}\left(\xi^{\prime}\right)=0
$$

The dispersion equation for the vorticity wave is obtained as for the entropy wave, namely,

$$
K_{v} \bar{M} \cos \left(\nu_{v}-\bar{\theta}\right)-1=0 .
$$

To first order, no pressure or entropy fluctuations are associated to this wave. Using Eq. (4) it can be seen that there is no density fluctuation. Eq. (1) can be reduced to

$$
\frac{\partial u^{\prime}}{\partial x}+\frac{\partial v^{\prime}}{\partial y}=0
$$

and using the wave-form of Eq. (7) for the vorticity wave, the resulting velocity field can be written as

$$
\frac{u^{\prime}}{\bar{c}}=-i \frac{\xi^{\prime}}{\omega} \frac{\sin \left(\nu_{v}\right)}{K_{v}}, \quad \frac{v^{\prime}}{\bar{c}}=i \frac{\xi^{\prime}}{\omega} \frac{\cos \left(\nu_{v}\right)}{K_{v}} .
$$


Using Eq. (6) these fluctuations can be written in terms of $w^{\prime}$ and $\theta^{\prime}$, namely

$$
\frac{w^{\prime}}{\bar{c}}=-i \frac{\xi^{\prime}}{\omega} \frac{\sin \left(\nu_{v}-\bar{\theta}\right)}{K_{v}}, \quad \theta^{\prime}=i \frac{\xi^{\prime}}{\omega} \frac{\cos \left(\nu_{v}-\bar{\theta}\right)}{\bar{M} K_{v}} .
$$

Using the non-dimensional form of the vorticity wave, $w^{v}=\xi / \omega$, the fluctuations of the primitive variables generated by this wave are

$$
\left\{\begin{array}{c}
s^{\prime} / C_{p} \\
w^{\prime} / \bar{c} \\
p^{\prime} / \gamma \bar{p} \\
\theta^{\prime}
\end{array}\right\}_{v}=\left\{\begin{array}{c}
0 \\
-i \frac{\sin \left(\nu_{v}-\bar{\theta}\right)}{K_{v}} \\
0 \\
i \frac{\cos \left(\nu_{v}-\bar{\theta}\right)}{\bar{M} K_{v}}
\end{array}\right\} w^{v} .
$$

\subsubsection{Acoustic waves}

As entropy and vorticity fluctuations are described by independent fluctuations, the acoustic waves generate a perturbated field which is irrotational and isentropic. Eqs.(1)-(4) can be combined to give the governing equation for the acoustic perturbations

$$
\left[\left(\frac{D}{D t}\right)^{2}-\bar{c}^{2}\left(\frac{\partial^{2}}{\partial x^{2}}+\frac{\partial^{2}}{\partial y^{2}}\right)\right]\left(\frac{p^{\prime}}{\gamma \bar{p}}\right)=0 .
$$

Using the waveform of Eq. (7) for the acoustic wave,

$$
w^{ \pm}=\left(\frac{p^{\prime}}{\gamma \bar{p}}\right)_{ \pm}=A_{ \pm} \exp \left[-i\left(\omega t-\mathbf{k}_{ \pm} \cdot \mathbf{x}\right)\right]
$$

the dispersion equation follows,

$$
\left(1-K_{ \pm} \bar{M} \cos \left(\nu_{ \pm}-\bar{\theta}\right)\right)^{2}-K_{ \pm}^{2}=0,
$$

where $K^{ \pm}$is the module of the non-dimensional wave vector. As the acoustic dispersion equation has two solutions, the subscript $( \pm)$ stands for the acoustic perturbation propagating upwards $(+)$ and downwards $(-)$. The equation can be written as a function of the axial and circumferential components $\left(K_{x}\right.$ and $\left.K_{y}\right)$ using Eq. (8),

$$
\left(1-K_{x, \pm} \bar{M} \cos \bar{\theta}-K_{y, \pm} \bar{M} \sin \bar{\theta}\right)^{2}-K_{x, \pm}^{2}-K_{y, \pm}^{2}=0 .
$$

When solving for Eq. (22), $K_{x}$ can be either real or complex valued. The real values are acoustic waves propagating without attenuation through the mean steady flow. Instead, complex values correspond to evanescent waves that cannot propagate in the flow considered. Solving for Eq. (22) yields the condition

$$
\left(1-K_{y} \bar{M} \sin \bar{\theta}\right)^{2}-\left(1-\bar{M}^{2} \sin ^{2} \bar{\theta}\right) K_{y}^{2}<0 .
$$

For a given mean flow, this relation gives a critical value of $K_{y}=\bar{c} k_{y} / \omega$. This shows that for any circumferential acoustic mode $k_{y}$ there exists a cut-off frequency above which the acoustic waves will not propagate. 
The fluctuation of primitive variables generated by the wave can be obtained from Eqs. (1)-(4) knowing that the wave is isentropic,

$$
\left\{\begin{array}{c}
s^{\prime} / C_{p} \\
w^{\prime} / \bar{c} \\
p^{\prime} / \gamma \bar{p} \\
\theta^{\prime}
\end{array}\right\}=\left\{\begin{array}{c}
0 \\
K_{ \pm} \cos \left(\nu_{ \pm}-\bar{\theta}\right) /\left[1-K_{ \pm} \bar{M} \cos \left(\nu_{ \pm}-\bar{\theta}\right)\right] \\
1 \\
K_{ \pm} \sin \left(\nu_{ \pm}-\bar{\theta}\right) /\left[\bar{M}\left(1-K_{ \pm} \bar{M} \cos \left(\nu_{ \pm}-\bar{\theta}\right)\right)\right]
\end{array}\right\} w^{ \pm}
$$

\subsubsection{Transformation Matrix}

The primitive fluctuating variables are written as a function of the four waves in a matrix form by adding the contribution of each wave, namely

$$
\left\{\begin{array}{c}
s^{\prime} / C_{p} \\
w^{\prime} / \bar{c} \\
p^{\prime} / \gamma \bar{p} \\
\theta^{\prime}
\end{array}\right\}=[\mathbf{M}] \cdot\left\{\begin{array}{c}
w^{s} \\
w^{v} \\
w^{+} \\
w^{-}
\end{array}\right\},
$$

where the matrix $[\mathbf{M}]$ is given by the combination of Eqs. (11), (18) and (24),

$$
[\mathbf{M}]=\left[\begin{array}{cccc}
1 & 0 & 0 & 0 \\
0 & -i \frac{\sin \left(\nu_{v}-\bar{\theta}\right)}{K_{v}} & \frac{K_{+} \cos \left(\nu_{+}-\bar{\theta}\right)}{\left(1-K_{+} \bar{M} \cos \left(\nu_{+}-\bar{\theta}\right)\right)} & \frac{K_{-} \cos \left(\nu_{-}-\bar{\theta}\right)}{\left(1-K_{-} \bar{M} \cos \left(\nu_{-}-\bar{\theta}\right)\right)} \\
0 & 0 & 1 & 1 \\
0 & i \frac{\cos \left(\nu_{v}-\bar{\theta}\right)}{K_{v} \bar{M}} & \frac{K_{+} \sin \left(\nu_{+}-\bar{\theta}\right)}{\bar{M}\left(1-K_{+} \bar{M} \cos \left(\nu_{+}-\bar{\theta}\right)\right)} & \frac{K_{-} \sin \left(\nu_{-}-\bar{\theta}\right)}{\bar{M}\left(1-K_{-} \bar{M} \cos \left(\nu_{-}-\bar{\theta}\right)\right)}
\end{array}\right] .
$$

\subsection{Matching conditions through the blade row}

Since the blade row is assumed to be axially compact, the upstream and downstream flow can be related through matching conditions. These conditions are obtained using the mass, energy and entropy conservation. As energy is not conserved through rotating blades, the study of the stator vane and of the rotor blade should be done separately.

\subsubsection{Stator vane}

For the stator vane the conservation equations used by Cumpsty and Marble read,

$$
\left(\frac{\dot{m^{\prime}}}{\dot{m}}\right)_{1}=\left(\frac{\dot{m^{\prime}}}{\dot{m}}\right)_{2}, \quad\left(\frac{T_{t}^{\prime}}{T_{t}}\right)_{1}=\left(\frac{T_{t}^{\prime}}{T_{t}}\right)_{2}, \quad\left(\frac{s^{\prime}}{C_{p}}\right)_{1}=\left(\frac{s^{\prime}}{C_{p}}\right)_{2} .
$$

These equations are completed with the Kutta condition at the outlet of the blade $\left(\theta_{2}^{\prime}=0\right)$, which can be written in a more general form as,

$$
\theta_{2}^{\prime}=\beta \theta_{1}^{\prime} \text {. }
$$


Subscripts 1 and 2 represent the flow upstream and downstream of the blade row as shown in Fig. 1. These equations should be written as a function of the primitive variables chosen before $\left(s^{\prime}, p^{\prime}, w^{\prime}\right.$ and $\left.\theta^{\prime}\right)$. This gives,

$$
\begin{aligned}
& \left(\frac{\dot{m}^{\prime}}{\overline{\dot{m}}}\right)=\frac{p^{\prime}}{\gamma \bar{p}}+\frac{1}{\bar{M}} \frac{w^{\prime}}{\bar{c}}-\theta^{\prime} \tan \bar{\theta} \\
& \left(\frac{T_{t}^{\prime}}{T_{t}}\right)=\frac{1}{1+\frac{(\gamma-1)}{2} \bar{M}^{2}}\left[(\gamma-1) \frac{p^{\prime}}{\gamma \bar{p}}+\frac{s^{\prime}}{C_{p}}+(\gamma-1) \bar{M} \frac{w^{\prime}}{\bar{c}}\right],
\end{aligned}
$$

Using Eqs. (27)-(28), and writing them as a function of the primitive variables using Eqs. (29)-(30), the jump conditions are obtained, which may be written in a matrix form,

$$
\left[\mathbf{E}_{1}\right] \cdot\left\{\begin{array}{c}
s^{\prime} / C_{p} \\
w^{\prime} / \bar{c} \\
p^{\prime} / \gamma \bar{p} \\
\theta^{\prime}
\end{array}\right\}_{1}=\left[\mathbf{E}_{2}\right] \cdot\left\{\begin{array}{c}
s^{\prime} / C_{p} \\
w^{\prime} / \bar{c} \\
p^{\prime} / \gamma \bar{p} \\
\theta^{\prime}
\end{array}\right\}_{2}
$$

with $\left[\mathbf{E}_{1}\right]$ and $\left[\mathbf{E}_{2}\right]$ defined as,

$$
\left[\mathbf{E}_{1}\right]=\left[\begin{array}{cccc}
1 & 0 & 0 & 0 \\
-1 & \frac{1}{\bar{M}_{1}} & 1 & -\tan \bar{\theta}_{1} \\
\frac{\mu_{1}}{\gamma-1} & \mu_{1} \bar{M}_{1} & \mu_{1} & 0 \\
0 & 0 & 0 & \beta
\end{array}\right], \quad\left[\mathbf{E}_{2}\right]=\left[\begin{array}{cccc}
1 & 0 & 0 & 0 \\
-1 & \frac{1}{\bar{M}_{2}} & 1 & -\tan \bar{\theta}_{2} \\
\frac{\mu_{2}}{\gamma-1} & \mu_{2} \bar{M}_{2} & \mu_{2} & 0 \\
0 & 0 & 0 & 1
\end{array}\right],
$$

where $\mu=1 /\left[1+(\gamma-1) \bar{M}^{2} / 2\right]$.

\subsubsection{Rotor blade}

For the rotor blade, the energy equation is no longer conserved through the interface. Instead, the rothalpy can be used as a conserved variable through the turbomachine. The rothalpy is defined as,

$$
I=h_{t}-U v
$$

where $h_{t}$ is the specific total enthalpy, $U$ is the rotating speed of the blade and $v$ the circumferential component of the absolute speed. For the rotating speed, a different value will be considered at the inlet and at the outlet for sake of generality, though in most applications $U_{1}=U_{2}$. The conservation of rothalpy can be easily shown using the energy equation and the Euler equation for turbomachines, namely,

$$
\Delta h_{t}=\tau=\Delta(U v)=U_{2} \cdot v_{2}-U_{1} \cdot v_{1} .
$$

Considering small perturbations, a generalized form of the energy equation can be written as,

$$
\frac{I_{1}^{\prime}}{\bar{I}_{1}}=\frac{I_{2}^{\prime}}{\bar{I}_{2}},
$$


with

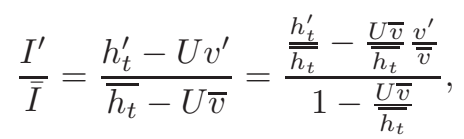

and defining the parameter $\zeta$ as

$$
\zeta=\frac{U \bar{v}}{\overline{h_{t}}},
$$

Eq. (36) can be re-written,

$$
\frac{I^{\prime}}{\bar{I}}=\frac{1}{1-\zeta}\left[\frac{T_{t}^{\prime}}{\overline{T_{t}}}-\zeta\left(\frac{1}{\bar{M}} \frac{w^{\prime}}{\bar{c}}+\frac{\theta^{\prime}}{\tan \bar{\theta}}\right)\right] .
$$

It can be seen that for $U=0, \zeta=0$ and the conservation of the total temperature used for the stator is recovered. Eq. (35) therefore replaces the second equation of Eq. (27) when considering rotating blades.

\subsection{Solution of the equations}

The matrix system is used to solve the problem imposing the correct boundary conditions. These are, in the subsonic case, $w^{+}, w^{s}$ and $w^{v}$ at the inlet, and $w^{-}$at the outlet of the blade row. The waves coming from the combustion chamber must be first decomposed in modes $K_{y}$ and in frequencies $\omega$. The propagation equations is solved for each pair $\left(k_{y}, \omega\right)$. For a given pair $\left(k_{y}, \omega\right)$, the dispersion equations can be used to obtain the value of the wave vector $\mathbf{K}$ for each wave. Using this information, the matrix $\mathbf{M}$ can then be calculated on both sides of the blade row.

For a single blade row, Eq. (31) can be written as a function of the waves using Eq. (25),

$$
\underbrace{\left[\mathbf{E}_{1}\right] \cdot\left[\mathbf{M}_{1}\right]}_{\left[\mathbf{B}_{1}\right]} \cdot\left\{\begin{array}{c}
w^{s} \\
w^{v} \\
w^{+} \\
w^{-}
\end{array}\right\}=\underbrace{\left[\mathbf{E}_{2}\right] \cdot\left[\mathbf{M}_{2}\right]}_{\left[\mathbf{B}_{2}\right]} \cdot\left\{\begin{array}{c}
w^{s} \\
w^{v} \\
w^{+} \\
w^{-}
\end{array}\right\}_{2} .
$$

The fourth column of matrices $\left[\mathbf{B}_{1}\right]$ and $\left[\mathbf{B}_{2}\right]$ should be permuted (and changed signs) in order to have a matrix system with the imposed waves on the right hand side of the equations and the unknowns on the left hand side. The resulting matrices, named $\left[\mathbf{A}_{i n}\right]$ and $\left[\mathbf{A}_{\text {out }}\right]$ give the system of equations,

$$
\left[\mathbf{A}_{\text {out }}\right] \cdot\left\{\begin{array}{c}
w_{2}^{s} \\
w_{2}^{v} \\
w_{2}^{+} \\
w_{1}^{-}
\end{array}\right\}=\left[\mathbf{A}_{\text {in }}\right] \cdot\left\{\begin{array}{c}
w_{1}^{s} \\
w_{1}^{v} \\
w_{1}^{+} \\
w_{2}^{-}
\end{array}\right\},
$$

which can be inverted to obtain the out-coming waves.

For the case of multiple blades, the phase-shift of the waves through the blade-row spacing is taken into account. This is done through the matrix $[\mathbf{T}]$, in which the diagonal terms are the phase-shift of the waves given by $\exp \left[+i k_{x, \phi} \cdot L\right]$, where $L$ is the distance between rows and $\phi$ the considered wave. 
Consider $\mathbf{V}_{u}^{i}$ and $\mathbf{V}_{d}^{i}$ the vector of waves upstream and downstream the $i$-th blade row. Eq. (39) relates both sides through

$$
\left[\mathbf{B}_{1}^{i}\right] \cdot \mathbf{V}_{u}^{i}=\left[\mathbf{B}_{2}^{i}\right] \cdot \mathbf{V}_{d}^{i}
$$

The downstream vector of waves can be related to the following blade row through $\mathbf{V}_{u}^{i+1}=\left[\mathbf{T}^{i}\right] \cdot \mathbf{V}_{d}^{i}$. Combining the successive stages of the considered turbine, a relation between the inlet and the outlet waves of the turbomachine can be written in a matrix form and finally permuted to obtain a system of equations as in Eq. (40), which can be inverted to obtain the out-coming waves.

\section{Methodology of Chorus}

The method developed to compute combustion noise is based on LES simulations of the combustion chamber, combined with analytical methods to propagate the waves through the turbine stages and to obtain the acoustic power at the outlet of the aero-engine as done in [7]. To combine the numerical simulations with the analytical model, a post-processing of the primitive variables at the outlet of the combustion chamber has to be performed. The method can be divided in six steps as shown in Fig. 2 .

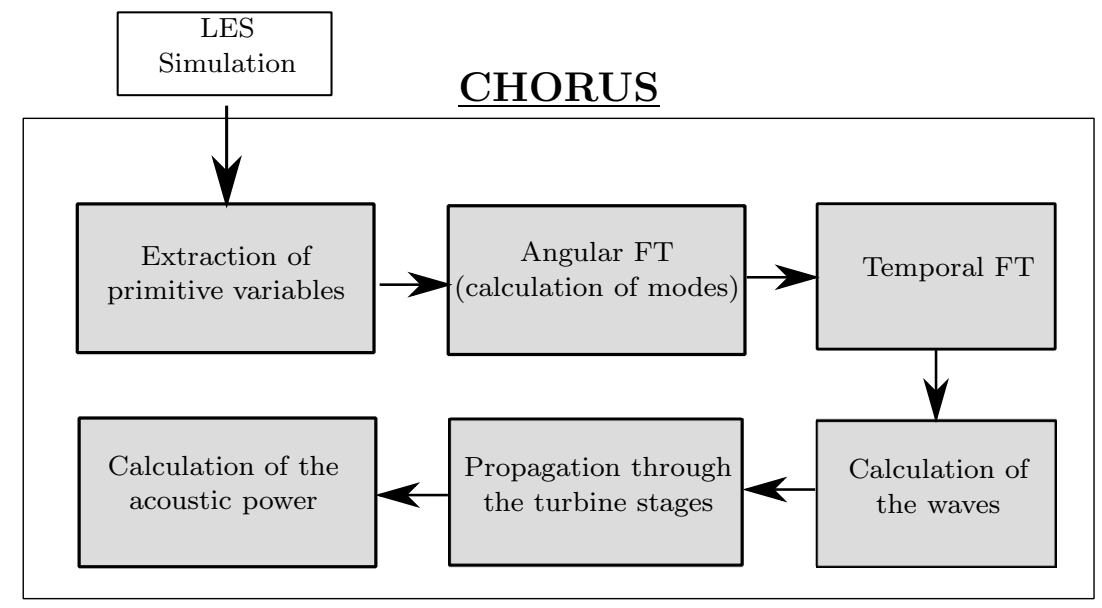

Figure 2. Schema of the procedure of Chorus to calculate the noise generated by the combustion chamber

The primitive variables are first extracted from the LES simulation at an outlet plane, at a distance of about one chord upstream from the High Pressure Turbine. These primitive variables are, in a 3D configuration, $p^{\prime}, s^{\prime}$ and $\mathbf{w}^{\prime}$, which are then projected in a polar coordinate system and averaged in the radial direction of the annular combustion chamber to obtain a two-dimensional set of fluctuating primitive variables. An angular Fourier transform (FT) followed by a Fourier transform in the time domain are performed to decompose the fluctuations in harmonic modes. The Welch method [8] is used to perform the Fourier transform over time: the temporal signal is split in $N$ segments with an overlapping region. This allows a smoothing of the signal in the frequency domain. Using the dispersion equations for each wave (Eqs. (10), (14) and (22)), and Eq. (25) the Cumpsty and Marble method described in Section 2 is then applied to obtain the waves at the outlet of the aero-engine. 


\section{Validation of the analytical model}

The analytical model is based on two main hypothesis. First the blade geometry is considered to be two-dimensional. Secondly, the model considers the blade spacing to be short and the blades to be axially compact compared to the wavelength of the perturbations. This last hypothesis is the most restrictive and is valid for the low frequency range only. Even though combustion noise is significant mainly at low frequencies, it is interesting to analyse the range of validity of the model in a simple case. To do so, numerical methods will be used to simulate the propagation though a simple stator row. A two-dimensional configuration will be used, neglecting therefore the possible three-dimensional effects.

In a first stage only plane waves are considered as shown in [9]. The numerical solver AVBP [2] is used to compute the propagation of $2 \mathrm{D}$ waves through a simple stator vane and a rotor blade (shown in Fig. 3) separately. A third order in space and fourth order in time scheme is used (TTG4A) to minimise dispersion and dissipation. A large turbulent Prandtl number is used in order to minimize dissipation of entropy waves by the turbulent structures downstream of the rotor blade. The mean Mach number of the rotor is illustrated in Fig. 4, showing an inlet Mach number of 0.653 and an outlet Mach number of 0.36.

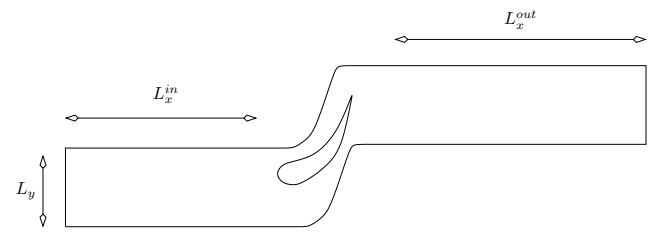

Figure 3. Sketch of the computational domain for the rotor

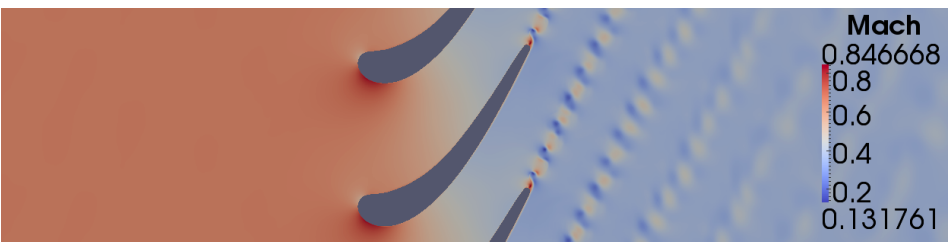

Figure 4. Mean Mach number through the rotor blades

The pulsated waves are imposed using NSCBC boundary conditions (Poinsot and Lele [10]). Several discrete frequencies are imposed in the same simulation. The pulsated wave follows the function

$$
f(t)=\sum_{k=1}^{N} \sin \left(2 \pi f_{0} k t\right)
$$

with $N=50$ the number of frequencies pulsed and $f_{0}=100 \mathrm{~Hz}$ the fundamental frequency. The regions at the inlet and at the outlet of the computational domain are used to perform the post-processing of the solutions and obtain the transfer functions of the stator. The results are shown in Figs. 5 and 6 , corresponding to the upstream and downstream propagating acoustic waves generated by an acoustic and an entropy perturbation at the inlet respectively. For the rotor, the results are shown in Fig. 7, where a downstream propagating acoustic wave is propagated through the rotor.

Results show a good agreement for the low frequency range both for the stator and the rotor, except for the transmission of acoustic waves through the rotor. Higher frequencies are less accurately predicted, though it is known that combustion noise acts mostly in the low frequency range. In the case of the rotor, the transmission coefficient obtained numerically differs from the analytical one. 


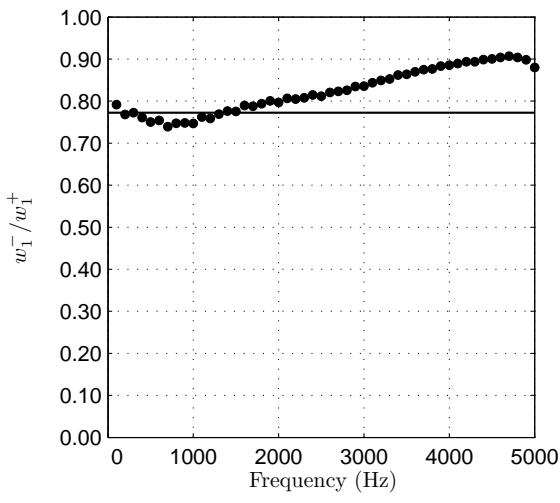

(a) Reflected wave

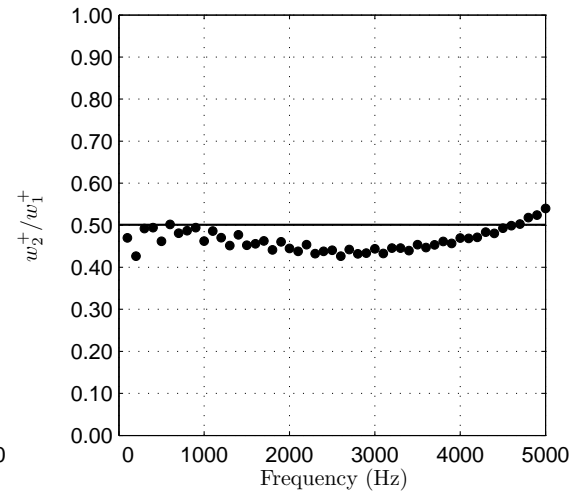

(b) Transmitted wave

Figure 5. Response of a stator vane to an acoustic perturbation at the inlet. Theory $(-)$, simulation $(\bullet)$.

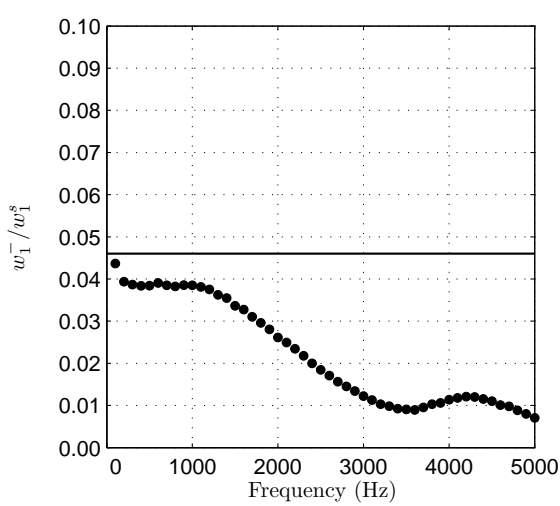

(a) Acoustic wave at the inlet

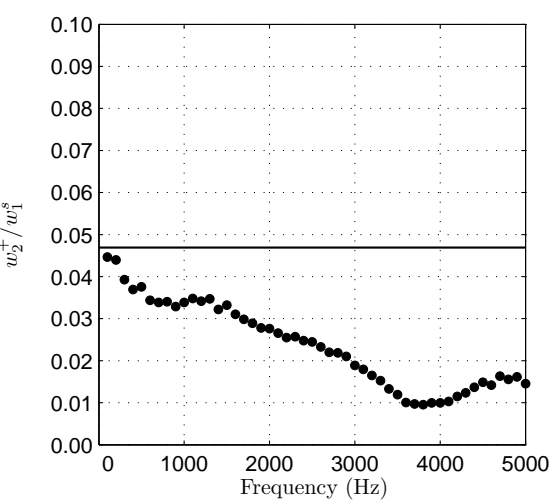

(b) Acoustic wave at the outlet

Figure 6. Response of a stator vane to an entropy perturbation. Theory $(-)$, simulation $(\bullet)$.

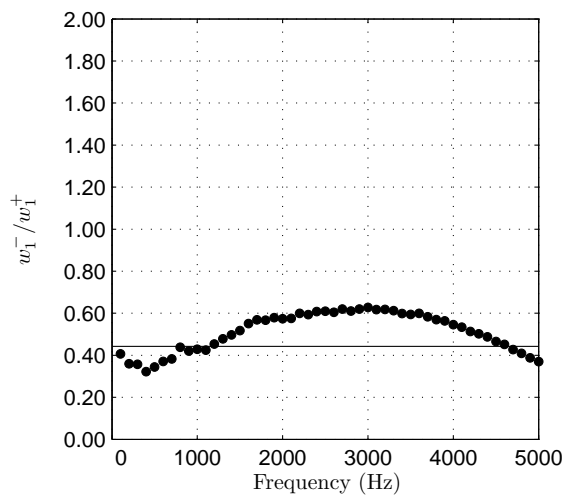

(a) Reflected wave

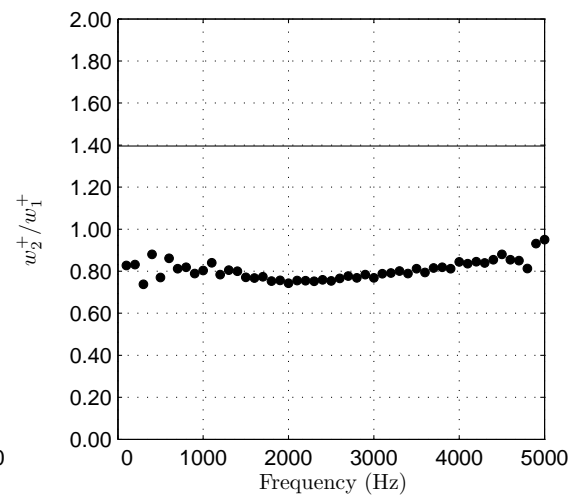

(b) Transmitted wave

Figure 7. Response of a rotor blade to an acoustic perturbation. Theory $(-)$, simulation $(\bullet)$.

\section{Conclusions}

A methodology to compute combustion noise has been implemented. The method, combining LES simulations with analytical models, allows the user1to calculate the combustion-generated noise at the 


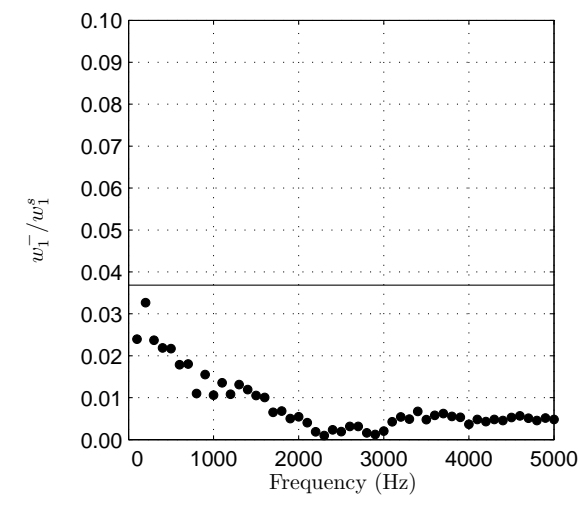

(a) Acoustic wave at the inlet

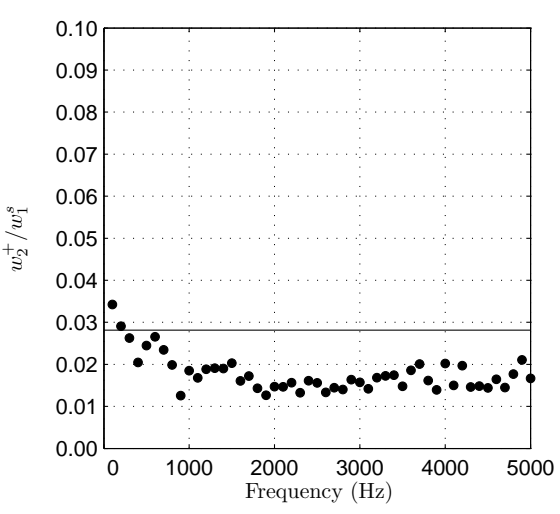

(b) Acoustic wave at the outlet

Figure 8. Response of a rotor blade to an entropy perturbation. Theory $(-)$, simulation $(\bullet)$.

outlet of the aero-engine. The computed noise is the total contribution of the different mechanisms generating combustion noise (direct and indirect), but the method differentiates both mechanisms, making it possible to compute the contribution of each one separately. This makes it possible to compute the contribution of each noise term in a real combustion-chamber configuration.

\section{References}

[1] F. E. Marble and S. Candel. Acoustic disturbances from gas nonuniformities convected through a nozzle. J. Sound Vib., 55:225-243, 1977.

[2] T. Schønfeld and M. Rudgyard. Steady and unsteady flows simulations using the hybrid flow solver AVBP. AIAA Journal, 37(11):1378-1385, 1999.

[3] N. A. Cumpsty and F. E. Marble. The interaction of entropy fluctuations with turbine blade rows; a mechanism of turbojet engine noise. Proc. R. Soc. Lond. A, 357:323-344, 1977.

[4] F. Bake, C. Richter, B. Muhlbauer, N. Kings, I.Rohle, F.Thiele, and B.Noll. The entropy wave generator (EWG): a reference case on entropy noise. J. Sound Vib., pages 574-598, 2009.

[5] M. Leyko, F. Nicoud, and T. Poinsot. Comparison of direct and indirect combustion noise mechanisms in a model combustor. AIAA Journal, 47(11):2709-2716, November 2009.

[6] M. Leyko, S. Moreau, F. Nicoud, and T. Poinsot. Numerical and analytical modelling of entropy noise in a supersonic nozzle with a shock. J. Sound Vib., doi:10.1016/j.jsv.2011.01.025, 2011.

[7] M. Leyko. Mise en oeuvre et analyse de calculs aéroacoustiques de type SGE pour la prévision du bruit de chambres de combustion aéronautiques. PhD thesis, INP Toulouse, 2010.

[8] D. G. Childers. Modern Spectrum Analysis. IEEE Press, 1978.

[9] M. Leyko, S. Moreau, F. Nicoud, and T. Poinsot. Waves transmission and generation in turbine stages in a combustionnoise framework. In 16th AIAA/CEAS AeroAcoustics Conference, 2010.

[10] T. Poinsot and S. Lele. Boundary conditions for direct simulations of compressible viscous flows. J. Comput. Phys. , 101(1):104-129, 1992. 